PROCEEDINGS OF THE

AMERICAN MATHEMATICAL SOCIETY

Volume 126, Number 8, August 1998, Pages 2505-2506

S 0002-9939(98)04698-X

\title{
HYPERSURFACES WITH POSITIVE PRINCIPAL CURVATURES IN SYMMETRIC SPACES
}

\author{
JAIME RIPOLL
}

(Communicated by Christopher Croke)

A classical result in differential geometry, known as Hadamard's theorem, establishes that a compact connected surface in Euclidean space whose principal curvatures are everywhere positive is the boundary of a convex body. In particular, the surface is diffeomorphic to a sphere $([\mathrm{H}])$. We present here, using a simple observation as proof, a partial extension of this theorem to immersions of arbitrary codimension and to other spaces than the Euclidean one, as symmetric spaces of noncompact type.

Let $M^{n}$ and $N^{n+k}$ be Riemannian manifolds of dimensions $n$ and $n+k, n \geq$ $2, k \geq 1, M$ compact, connected, and let $\phi: M \rightarrow N$ be an isometric immersion. Denote by $N(M)$ the unit normal bundle of $\phi$, namely

$$
N(M)=\left\{(p, \eta) \mid p \in M, \eta \in T_{\phi(p)} N, \eta \perp \phi_{*}\left(T_{p} M\right),\|\eta\|=1\right\} .
$$

We denote by $N^{*}(M)$ the subbundle of $N(M)$ consisting of the pairs $(p, \eta)$ such that the 2nd fundamental form $A$ of $\phi$ with respect to $\eta$ at $p$, that is, $A_{\eta}(v)=$ $\left(\nabla_{\phi_{*} v} \eta\right)^{\mathrm{T}}, v \in T_{p} M$, has positive eigenvalues, $\nabla$ being the Riemannian connection of $N$ and ( $)^{\mathrm{T}}$ the orthogonal projection to $\phi_{*}\left(T_{p} M\right)$. We prove:

Proposition. According to the above notation, assume the existence of $n+1$ Killing fields $X_{1}, \ldots, X_{n+1}$ in $N$ which are linearly independent on $\phi(M)$ and a global section $\eta: M \rightarrow N^{*}(M)$ such that $\eta(p) \in \operatorname{span}\left\{X_{1}, \ldots, X_{n+1}\right\}$ for all $p \in M$. Then $M$ is diffeomorphic to an $n$-dimensional sphere.

We have the following immediate consequences:

Corollary 1. Let $N$ be a homogeneous manifold with an invariant metric. Then there is an open dense subset $U$ of $N$ such that any immersed compact, connected hypersurface of $U$ whose principal curvatures are positive is diffeomorphic to a sphere.

Corollary 2. Let $G$ be a Lie group with a left invariant metric. Then, any compact connected immersed hypersurface of $G$ whose principal curvatures are positive is diffeomorphic to a sphere.

We remark that Corollary 2 applies, for example, to a symmetric space $G / K$ of noncompact type since, by the Iwasawa decomposition $G=K A N, G / K$ is isometric to the solvable Lie group $S=A N$ with a certain left invariant metric.

Received by the editors May 9, 1997.

1991 Mathematics Subject Classification. Primary 53C42; Secondary 53C30.

Key words and phrases. Immersions, positive principal curvatures, symmetric spaces.

This research was partially supported by CNPq (Brazil). 
Proof of the proposition. Given $p \in M$, set

$$
E_{p}=\left\{a_{1} X_{1}(\phi(p))+\cdots+a_{n+1} X_{n+1}(\phi(p)) \mid a_{1}^{2}+\cdots+a_{n+1}^{2}=1\right\} .
$$

From the hypothesis, there is a unit normal vector field $\eta$ to $\phi$ such that the 2nd fundamental form of $M$ with respect to $\eta$ is positive definite and $\eta(p) \in$ $\operatorname{span}\left\{X_{1}(\phi(p)), \ldots, X_{n+1}(\phi(p))\right\}$, for all $p \in M$. There exists a differentiable map $f: M \rightarrow \mathbb{R}^{+}$such that $f(p) \eta(p) \in E_{p}$, for all $p \in M$. We define a map $\gamma: M \rightarrow S^{n}$, where $S^{n}$ is the unit sphere centered at the origin in $\mathbb{R}^{n+1}$, by setting

$$
\gamma(p)=\left(a_{1}(p), \ldots, a_{n+1}(p)\right)
$$

$$
f(p) \eta(p)=a_{1}(p) X_{1}(\phi(p))+\cdots+a_{n+1}(p) X_{n+1}(\phi(p)), \quad p \in M .
$$

We claim that $\gamma$ is a diffeomorphism. Clearly $\gamma$ is a differentiable map. Choose $p \in M$ and let $v \in T_{p} M$ such that $d \gamma_{p}(v)=0$. From (1), we have $d\left(a_{j}\right)_{p}(v)=$ $0, j=1, \ldots, n+1$.

Taking the covariant derivative of (2) with respect to $v$ we therefore obtain

$$
d f_{p}(v) \eta(p)+f(p) \nabla_{v} \eta(p)=a_{1}(p) \nabla_{v} X_{1}+\cdots+a_{n+1}(p) \nabla_{v} X_{n+1} .
$$

Taking the inner product of both sides with $v$, since the $X_{j}$ are Killing fields, we obtain $f(p)\left\langle\nabla_{v} \eta, v\right\rangle=0$, which implies $v=0$, since $\eta \in N^{*}(M)$. It follows that $\gamma$ is a local diffeomorphism so that, since it goes into the sphere and $M$ is compact, $\gamma$ is a global diffeomorphism.

\section{ACKNOWLEDGEMENT}

I would like to thank J. Eschenburg and the referee for worthy mathematical comments concerning the results of this note.

\section{REFERENCES}

[H] M. Hadamard, Sur certaines proprietés des trajectoires en Dynamique, Journal de Math. (5 ${ }^{\mathrm{a}}$. série), Tome III, Fasc. IV, 1987.

Universidade Federal do R. G. do Sul, Instituto de Matemática, Av. Bento Gonçalves 9500, 91540-000 Porto Alegre - RS, Brazil

E-mail address: ripoll@athena.mat.ufrgs.br 